\title{
Prognostic Significance of Ratio of Metastatic Lymph Nodes (MLNR) to Total Lymph Nodes Harvested in Squamous Cell Carcinoma of Esophagus
}

\author{
Vikas Sharma ${ }^{1}$, S Krishnamurthy ${ }^{2}$ \\ ${ }^{1}$ Mch Resident, Department of Surgical Oncology, Kidwai Memorial Institute of Oncology, Bangalore, Karnataka, India- 560029 \\ ${ }^{2}$ Professor \& Head, Department of Surgical Oncology, Kidwai Memorial Institute of Oncology, Bangalore, Karnataka, India- 560029
}

\begin{abstract}
Introduction: Esophageal cancer is an aggressive disease with features of poor prognosis and increasing incidence. Survival varies widely according to cancer location, extent, spread and treatment. Presence of lymph node (LN) metastases in patients with esophageal cancer has important prognostic implications. Despite the prognostic significance of identifying $L N$ metastases in patients with esophageal cancer, the minimum number of LNs that need to be removed during surgery is controversial. Recently Metastatic Lymph Node Ratio (MLNR) is considered as an important prognostic factor. Aim and objectives: To know the prognostic significance of MLNR in patients with carcinoma of esophagus and to compare TNM staging based OS with MLNR based OS, and to study if MLNR based OS is significant as compared to TNM based OS. Material and methods: This study is an experimental, Prospective and retrospective clinical study during a period of 3 years from between January 2012 and December 2015 at a tertiary cancer center in South India. All patients were staged in accordance to the $7^{\text {th }}$ edition of AJCC. All patients were operated by Trans Hiatal Esophagectomy (THE). Patients were grouped according to $N$ stage which was further sub grouped as N0, N1, N2 and N3 depending on number of positive LN. Patients were also grouped according to MLNR into three subgroups as LO, L1, and L2, depending on MLNR. $O S$ was calculated in all subgroups of $N$ and all subgroups of $L$. Result: results of this study showed that MLNR as an important prognostic factor for $O S$ of esophagus cancer. OS according to TNM staging was found to significant among all four subgroups of $N$ (NO, N1, N2, N3). OS according to MLNR was also found to significant among all three subgroups L (LO, L1, L2). Comparison of OS in TNM and MLNR groups was found to be significant. Different patients in the same TNM group showed different OS based on their MLNR. Hence MLNR can be considered as an important prognostic factor in determining OS in esophagus cancer. Conclusion: MLNR may be considered as an important prognostic factor in prediction of survival of patients with esophagus cancer. A modified classification should be considered for higher accuracy in relation to treatment and survival of patients.
\end{abstract}

Keywords: Esophagus carcinoma, MLNR, overall survival, carcinoma, esophagus, MLNR

\section{Introduction}

Esophageal cancer (EC) is known as one of the most deadly malignancies affecting humans. Esophageal cancer is an aggressive disease with features of poor prognosis and increasing incidence. It is an important cause of cancer related deaths in the world. Five year survival rate is estimated only about $10 \%$ which ranges from $15 \%$ to $40 \%$ after surgery. EC presents with two distinct histopathologic types: squamous cell carcinoma and adenocarcinoma. The occurrence of type of cancer occurs in a given patient depends on several factors like lifestyle, socioeconomic status, and environmental factors. ${ }^{1}$

Esophageal carcinoma affects more than 450000 people worldwide and the incidence is rapidly increasing. EC is four times more common and slightly morelethal in men than in women. In recent decades, a profound increase in incidence rates of adenocarcinoma is seen in United States, along with many other Western countries. Whereas squamous cell carcinoma continues to predominate worldwide. $^{2}$

Worldwide predominant histologic type of esophageal cancer is SCC, more common among black population, with a peak in seventh decade of life. An esophageal "cancer belt," primarily squamous cell cancers, is the area extending from northeast China to the Middle East. Most adenocarcinomas arise in the distal part of esophagus, are more common in white men. Barrett's esophagus is commonest precancerous condition, risk of developing cancer in patients with Barrett's esophagus is 50 to 100 times higher than general population.

Tobacco and alcohol consumption are known to be primary causes of squamous cell carcinomas, whereas obesity is one of the emerging risk factor for esophageal adenocarcinoma. Barrett's esophagus is another clearly recognized risk factor for EC .Cigarettes, alcohol, red meat, hookah smoking, nass use (a chewing tobacco product), drinking hot tea, opium consumption, poor oral health, low intake of fresh fruit and vegetables, and low socioeconomic status also have been associated with a higher risk of esophageal SCC. ${ }^{4}$

Patients may present with various symptoms depending on progress of the disease. Dysphagia, weight loss, symptomatic gastroesophageal reflux,odynophagia, dull retrosternal pain, bone pain secondary to bone metastases, and cough or hoarseness secondary to paratracheal nodal or recurrent laryngeal nerveinvolvement, are various presentations.Diagnosis is made by upper endoscopy and a biopsy to establish a tissue diagnosis.Ca esophagus metastasises via blood, lymphatics and locally. The sentinel

Volume 6 Issue 12, December 2017 


\section{International Journal of Science and Research (IJSR) \\ ISSN (Online): 2319-7064}

Index Copernicus Value (2016): 79.57 | Impact Factor (2015): 6.391

node is the first lymphatic drainage area from the primary tumor, and could be the first site of micrometastasis. Esophageal cancer metastasizes to different regional and distant lymph nodes depending on the primary site,and early esophageal cancer invading the muscularis mucosae may have more than one lymph node metastasis. Various modes of treatment available depends onstage of the carcinoma, which includes Surveillance, Ablative Methods including Endoscopic Mucosal Resection and Minimally Invasive Esophagectomy, surgical Resection including Transhiatal Esophagectomy, Transthoracic Esophagectomy, Extended Esophagectomy, Adjuvant Therapy, Preoperative Chemotherapy. ${ }^{5}$

The presence of lymph node (LN) metastases in patients with esophageal cancer has important prognostic implications. Despite the prognostic significance of identifyingLN metastases in patients with esophageal cancer, the minimum number of LNs that need to be removed during surgery is controversial. There have been two primary opinions in recent years. Some agree with a three-field lymphadenectomy and hold that it is essential to achieve improved postoperative survival by resecting adequate lymph nodes in the neck because cervical lymph node metastases have been documented as approximately $20 \%$ to $40 \%$. Others argue that two-field lymphadenectomy is enough to dissect all the possible metastatic lymph nodes, including recurrent nerve chain lymph nodes from the superior mediastinum up to the neck, with less perioperative complications and the same outcome. A consistent lymphadenectomy strategy has yet to be established. ${ }^{6}$

Some recent studies have addressed staging issues in patients with esophageal carcinoma, including the prognostic significance of the extent of LN involvement the number of LNs examined, and the proportion of positive LNs. However, till date there is no clear cut recommendation about the extent of $\mathrm{LN}$ dissection and importance of ratio of of positive LN status on survival in EC. ${ }^{7}$.

Current study is aimed at deciding the prognostic significance of ratio of metastatic $\mathrm{LN}$ to total $\mathrm{LN}$ harvested during surgery in patients having SCC of esophagus.

\section{Aim \& Objectives}

1) To know the prognostic significance of ratio of metastatic lymph nodes to total lymph nodes harvested in patients with squamous cell carcinoma of esophagus.

2) To compare TNM staging based OS with MLNR based OS, and to study if MLNR based OS is significant as compared to TNM based OS

\section{Patients and Methods}

This is an experimental study( Prospective and retrospective clinical study). This study was undertaken based on a retrospective review of prospective data base of esophageal cancer patients treated at a tertiary cancer center in South India between January 2012 and December 2015. All patients were staged in accordance to the $7^{\text {th }}$ edition of American Joint Committee on Cancer (AJCC) cancer staging manual.

\section{Inclusion Criteria}

1) Patients willing to participate in the study.

2) Histologically confirmed SCC of the thoracic esophagus.

3) Patients with operable squamous cell carcinoma of esophagus operated in our institute.

4) Patients who Underwent curative surgery by Trans Hiatal esophagectomy(THE)

\section{Exclusion Criteria}

1) Inoperable/ Metastatic tumor

2) Adenocarcinoma of esophagus

3) Esophagogastric junction tumor, siewert type III

4) Tumors of upper third of esophagus and upper esophageal sphincter.

5) Adherence to the aorta $>90$ degree, invasion of the tracheo-bronchi, vertebrae etc.

Total of 87 patients were included in the study, Sample size was calculated with $5 \%$ level of significance and $80 \%$ power. All patients enrolled at our institute in the study duration and satisfying the inclusion criteria were included in the study. Patients were diagnosed with relevant investigations and underwent standard treatment as per institute's protocol. Patients and attendants were explained about the study. Informed consent was taken from those who agreed for participating in the study. Institute's Ethical committee approval was taken to perform the study. Patients were recruited both prospectively and retrospectively. For retrospective recruitment, data was collected from medical record department. All patients were also divided into four subgroups groups, according to TNM staging. $\mathrm{N} 0=$ without $\mathrm{LN}$ metastasis, N1= 1-2 LN metastasis, N2=3-6 LN metastasis, $\mathrm{N} 3=>7 \mathrm{LN}$ metastasis. The number of patients in N0,N1, N2 and N3 group were 45, 24, 15 and 3 respectively.Patients were divided into three subgroups, based on MLNR. L0= Patients with MLNR=0, L1= Patients with MLNR <0.1, and L2 = Patients with MLNR >0.1. The number of patients in L0, L1 and L2 group were 45, 18, and 24 respectively.

OS was calculated in patients of all four sub groups of TNM group and analysis was done to find out OS in all TNM subgroups was significant or not .

OS was calculated in patients of all three sub groups of MLNR group and analysis was done to find out OS in MLNR subgroups was significant or not.

OS of both TNM group and MLNR group was compared to find out whether MLNR is more important prognostic factor or not in relation to OS in cancer esophagus patients.

Statistical analysis was performed with the help of SPSS version 22software. Proportions were compared using the Chi-square test. Survival data was generated using life table methods. Differences in survival estimates were compared using log-rank test. Prognostic factors in the treatment groups were analyzed with the aid of Cox proportionate univariate and multivariate regression analysis. 


\section{Result}

\section{Patient Characteristics}

This study included 87 patients. $57(65.5 \%)$ patients were males and $30(34.5 \%)$ pt were females.

Age at diagnosis ranged from 41 to 74 years with median age of 62 years. Risk factor of smoking, tobacco and/or alcohol was present in $82 \%$ of patients.

\section{Tumor characteristics}

Most common location of the tumor was in midthoracic esophagus.

Most common T stage was T2 $(n=48)$, followed by T3 $(n=$ 33 ), followed by T1 ( $\mathrm{n}=4)$, followed by T4 ( $\mathrm{n}=2)$.

Most common N stage was N0 ( $\mathrm{n}=45)$, followed by N1 ( $\mathrm{n}=24$ ), followed by N3 ( $\mathrm{n}=15)$, followed by $\mathrm{N} 4(\mathrm{n}=3)$.

None of the patient had distant metastasis all patients were in M0 stage.
Adjuvant chemo or radiotherapy was given in 42 (48.2\%)patients.

Number of total lymph nodes resected ranged from 5 to 40 With an average number of 16.3 .

Number of total lymph nodes found to be metastatic, ranged from 0 to 9 .

\section{Survival characteristics}

\section{OS according to TNM staging}

Survival range of N0 was 31.5 month to 35.6 month with median of 33.6 month,

Survival range of N1 was 22.1 monthto 24.9 month with median of 23.5 month,

Survival range of N2 was 13.1 monthto 15.7 month with median of 14.4 month.

Median survival of N3 was 9 month. On comparison of OS among all four groups it was found to be significant $(\mathrm{p}=$ .000) (Figure 1)

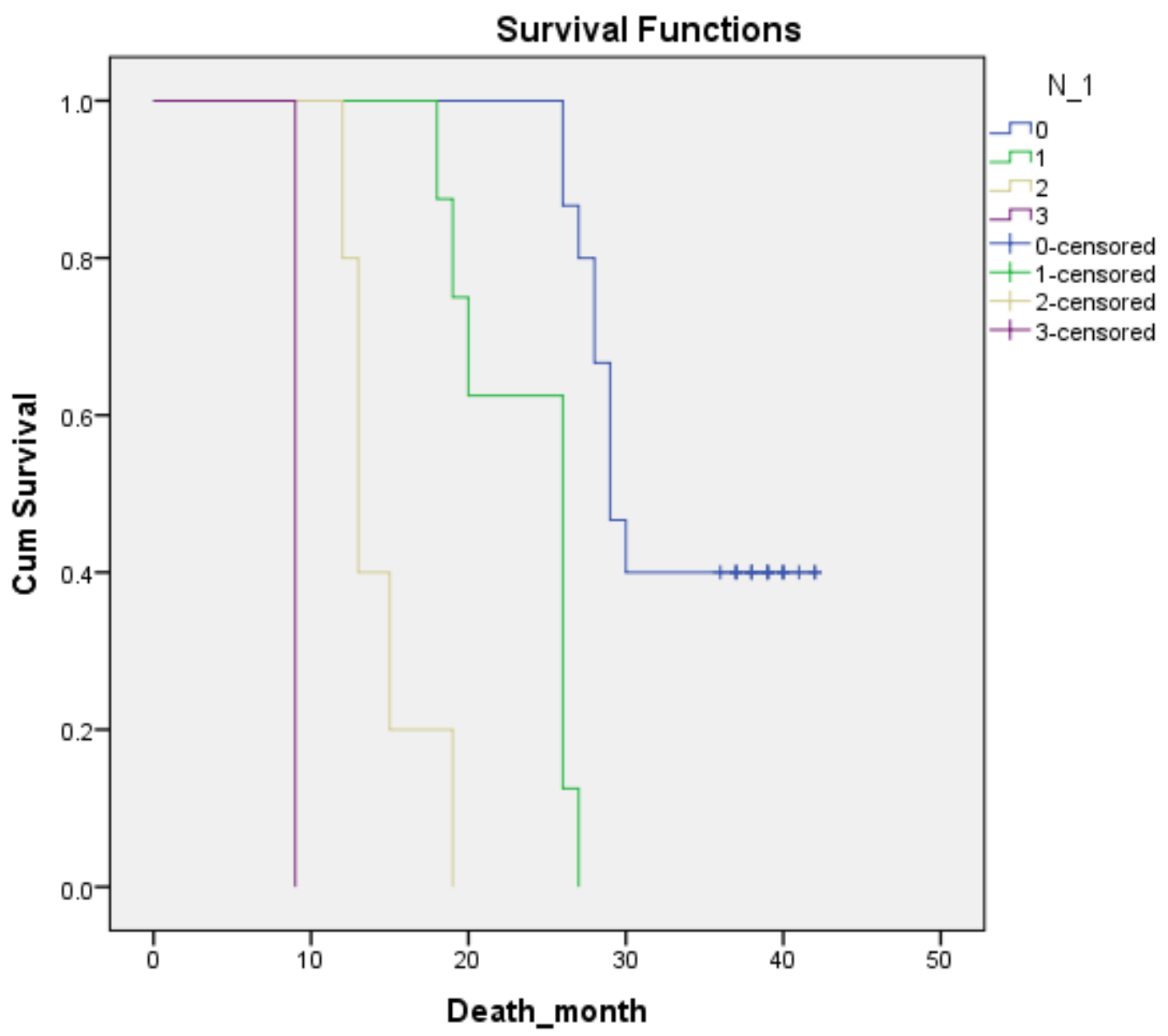

Figure 1: Showing OS in N subgroups

\section{OS according to MLNR}

Survival range of L0 group was 31.5 month to 35.6 with median of 33.6 month.

Survival range of L1 group was 21.1 month to 25.3 month with median of 23.2 month.
Survival range of L2 group was 14.1 month to 18.3 month with median of 16.2 month, on comparison of OS among all three subgroups it was found to be significant $(p=.000)$ (Figure 2) 


\section{International Journal of Science and Research (IJSR) \\ ISSN (Online): 2319-7064}

Index Copernicus Value (2016): 79.57 | Impact Factor (2015): 6.391

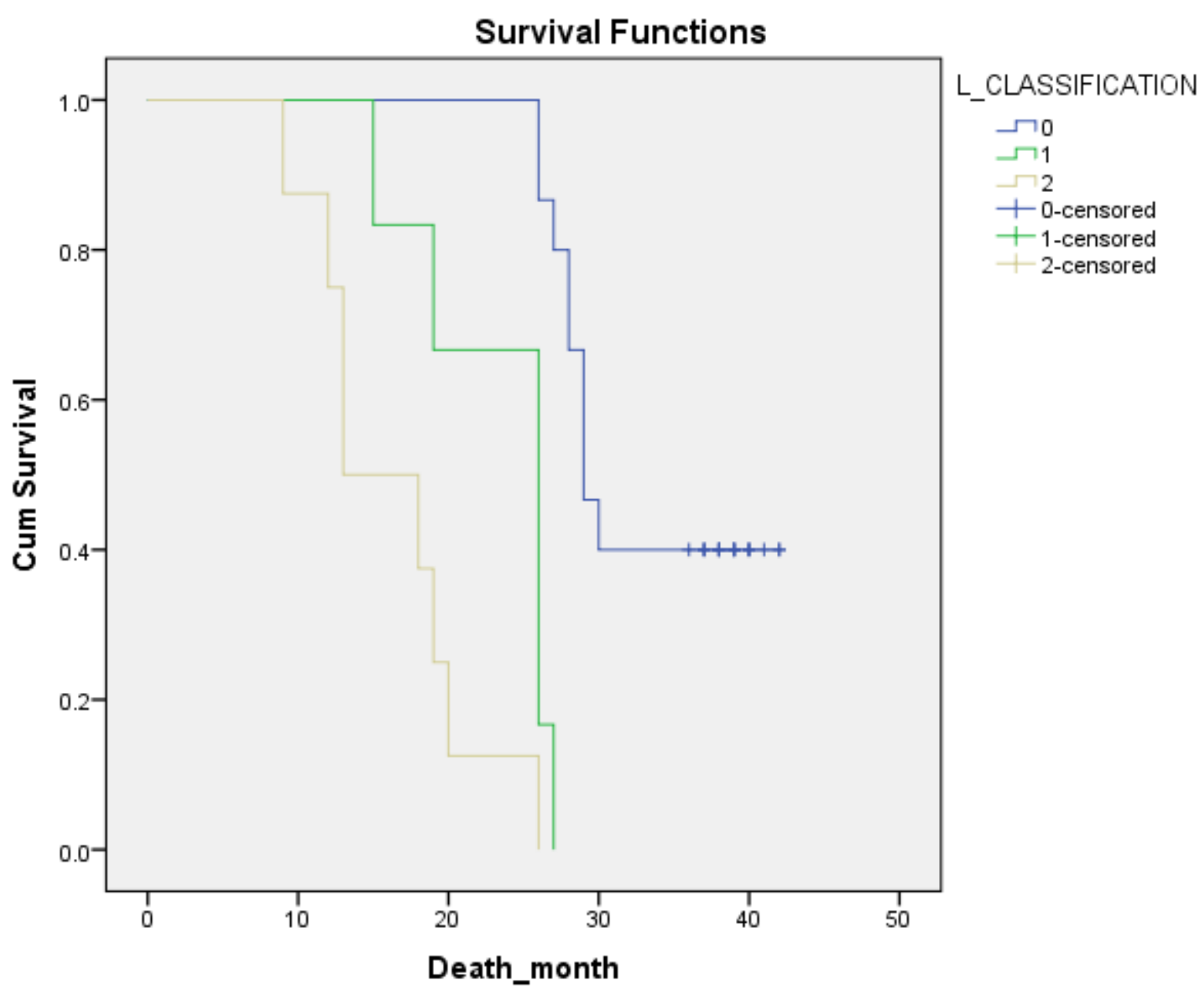

Figure 2: Showing OS in MLNR subgroups

Among total of 24 patients of $\mathrm{N} 1$ group 12 were in L1 group ( MLNR $\leq 0.1$ ) and 12 were in L2 group ( MLNR $>0.1$ ). Hence half of $\mathrm{N} 1$ patients were assigned to different prognostic group when grouped according to MLNR. Those patients in N1 group in which total number of LN harvested were less fell in to poor prognostic group (L2) with lesser OS as compared to those with higher number of total harvested LN which fell in to L1 group with significantly better OS $(\mathrm{p}=0.000)$. (Figure 3$)$

\begin{tabular}{|l|l|l|l|l|l|}
\hline \multicolumn{2}{|l|}{ N1 (n=24) } & \multicolumn{2}{l|}{ N2(n=15) } & \multicolumn{2}{l|}{ N3(n==3) } \\
\hline L1 & L2 & L1 & L2 & L1 & L2 \\
\hline 12 & 12 & 6 & 9 & 0 & 3 \\
\hline
\end{tabular}

Figure 3: Showing comparison between $\mathrm{N}$ and MLNR subgroups

Among total of 15 patients of N2 group 6 were in L1 group $($ MLNR $\leq 0.1)$ and 9 were in L2 group (MLNR >0.1). hence about two third of those patients in N2 group in which total number of LN harvested were less fell in to poor prognostic group (L2) with lesser OS as compared to about one third of those with higher number of total harvested LN which fell in to L1 group with significantly better OS $(\mathrm{p}=0.000)$.

All N3 patients fell in to L2 group (MLNR >0.1) suggesting similar prognosis irrespective of either nodal or MLNR grouping, may be because of smaller size of $\mathrm{N} 3$ patients.

\section{Discussion}

Esophageal cancer presents in advanced stage in many patients which is associated with poor outcomes. However appropriate treatment can not only improve the outcome but also reduce the morbidity.

Traditionally Surgical resection was the treatment of choice for carcinoma esophagus. Several improvements in the operative techniques and the extent of lympadenectomy have been done but the overall survival continues to remain poor. ${ }^{8}$

Various prognostic factors in carcinoma esophagus broadly includes patient factors, age at diagnosis, extent of disease, lymph node involvement and presence or absence of distant metastasis. However Lymph nodal involvement is known to be one of the most important prognostic factor in carcinoma esophagus. ${ }^{9}$

Cancer of Esophagus spreads via lymph nodes and esophagus has several number of lymph nodes draining to it. Number of lymph nodes may range from 6 to up to 23, and hence decision making on number of lymph nodes for adequate lymphadenectomy is confusing. Lac of standard criteria for adequacy of lymph node dissection contributes to the existence of question about cut off point for adequate lymphadenectomy since lymph node involvement represents a great impact on prognosis and survival, various

Volume 6 Issue 12, December 2017

\section{www.ijsr.net}

Licensed Under Creative Commons Attribution CC BY 


\section{International Journal of Science and Research (IJSR) \\ ISSN (Online): 2319-7064}

Index Copernicus Value (2016): 79.57 | Impact Factor (2015): 6.391

modifications in the treatment modalities are focused on resection of lymph node during surgery.Prognosis is directly related to increase in number of positive lymph nodes. Those patients having higher number of positive lymph nodes have poorer prognosis and overall survival as compared to those with lesser number of positive lymph nodes. ${ }^{10,11}$

Eloubeidi et al. conducted a study on patients of esophageal carcinoma. Tumor length, the number of involved lymph nodes, and the ratio of positive lymph nodes were noted among important prognostic factors. They concluded that increasing MLNR was associated with a poorer prognosis and a revised TNM classification system for patients with esophageal carcinoma might consider adding tumor length and number of positive lymph nodes as two important prognostic factors. ${ }^{12}$

In another study Nigro et al.Observed that the most important predictor of likelihood of 5-year survival and recurrent disease were presence and number of $\mathrm{LN}$ metastases and the ratio of involved to total $\mathrm{LN}$ removed. They concluded that patients with an MLNR $<0.1$ showed significantly better survival than those who had an MLNR $\geq$ $0.1 .^{13}$

Matthew Wilson B.A, et al. studied the Prognostic Significance of Lymph Node Metastases and Ratio in Esophageal Cancer.Perioperative mortality was $5.7 \%$. Median overall survival (OS) of the entire group was 22 months and 5-year OS was $27 \%$. The most significant prognostic factor for overall survival was the presence of positive LN $(\mathrm{p}=0.01)$. Additionally, patients with zero involved LN had a 5-year survival of 34\%, while patients with 1-3 positive LN and >3 positive LN had 5-year survival of $27 \%$ and $9 \%$, respectively $(p=0.01)$. Finally, an increasing ratio of positive to examined $\mathrm{LN}$ was linearly associated with a worsening 5 -year survival, $(\mathrm{p}=0.153)^{.14}$

He et al. studied Use of the Metastatic Lymph Node Ratio to Evaluate the Prognosis of Esophageal Cancer Patients with Node Metastasis Following Radical Esophagectomy.The median follow-up time was 41 months, and the 1-, 3- and 5year OS rates were $71.2 \%, 30.4 \%$, and $19.5 \%$, respectively. Univariate analysis showed that age, $\mathrm{pN}$ stage, and the MLR were prognostic factors for OS. Multivariate analysis indicated that a high MLR and advanced age were significant and independent risk factors for poor OS. ${ }^{15}$

Feng et al. in a retrospective analysis of 132 patients (>70 years) with esophageal SCC reported MLNR staging predicted survival similar to the 2010 AJCC N classification and felt that it should be considered an alternative to current $\mathrm{N}$ staging. They stated that all patients undergoing surgery for carcinoma esophagus should be staged according to MLNR because this more accurately predicted survival than current staging systems. ${ }^{16}$

Chen LQ et al demonstrates that the number of metastatic $\mathrm{LN}$ is an important prognostic factor, therefore, it should be considered when refining UICC-TNM classification for esophageal cancer. If the total number of LN removed during each esophagectomy is less than 6 , the occult positive regional $\mathrm{LN}$ might be missed, resulting in an inaccurate $\mathrm{N}$ classification and incorrect staging. Removal of $\geq \mathrm{LN}$ for each esophageal cancer

patient during esophagectomy recommended by UICC is rational and should be complied with. ${ }^{17}$

Greenstein AJ et al suggested that a higher LNR among patients with node-positive esophageal cancer is associated with worse survival.The study cohort included 838 esophageal cancer patients. Disease-specific survival rates decreased with higher LNR. Five-year disease-specific survival was $30 \%$ among patients with an $\mathrm{LNR}<$ or $=0.2$, compared with $16 \%$ and $13 \%$ for those with LNs of 0.21 to 0.5 and $>0.5$, respectively ( $\mathrm{p}<0.001)$. A higher LNR was independently associated with worse disease-specific survival. They recommended 18 nodes as the minimum number of resectable lymph nodes, whereas a consensus conference of the International Society for the Diseases of the Esophagus in 1995 suggested that accurate pathological staging of esophageal carcinoma requires resection of at least 15 nodes. $^{18,19}$

Our study shows that increasing MLNR is significantly associated with poorer prognosis. We compared MLNR separately in each $\mathrm{N}$ stage also. We found that all patients in N1 did not show similar OS, patients with high MLNR showed poorer prognosis as patients with low MLNR showed relatively better prognosis. Similar trend was observed in $\mathrm{N} 2$ and $\mathrm{N} 3$ patients also. Hence different patients in the same $\mathrm{N}$ group may have different $\mathrm{OS}$, depending on their MLNR. Our study strongly suggests the need of standardized and modified classification for esophagus cancer and MLNR may be included in the nodal classification.

\section{Conclusion}

MLNR may be considered as an important prognostic factor in prediction of survival of patients with esophagus cancer. The MLNR is more accurate prognostic factor as compared to TNM nodal stage, especially in patients with limited lymph node harvests. Hence a modified classification should be considered for higher accuracy in relation to treatment and survival of patients.

\section{References}

[1] Jemal A, Bray F, Center MM, Ferlay J, Ward E, Forman D. Globalcancer statistics. CA Cancer J Clin2011; 61: 69-90

[2] ennathur A, Gibson MK, Jobe BA, Luketich JD. Oesophagealcarcinoma. Lancet 2013; 381: 400-412

[3] Baquet CR, Commiskey P, Mack K, Meltzer S, Mishra SI.Esophageal cancer epidemiology in blacks and whites: racialand gender disparities in incidence, mortality, survival ratesand histology. J Natl Med Assoc2005; 97: 1471-1478

[4] Nigro JJ, DeMeester SR, Hagen JA, et al. Node status intransmural esophageal adenocarcinoma and outcome afteren bloc esophagectomy. J ThoracCardiovasc Surg. 1999; 117:960-968

[5] Mao WM, Zheng WH, Ling ZQ. Epidemiologic risk factorsfor esophageal cancer development. Asian Pac J Cancer Prev2011; 12: 2461-2466

\section{Volume 6 Issue 12, December 2017}




\section{International Journal of Science and Research (IJSR) \\ ISSN (Online): 2319-7064 \\ Index Copernicus Value (2016): 79.57 | Impact Factor (2015): 6.391}

[6] Gu Y, Swisher SG, Ajani JA, et al. The number of lymphnodes with metastasis predicts survival in patients withesophageal or esophagogastric junction adenocarcinomawho receive preoperative chemoradiation. Cancer. 2006;106:1017-1025.

[7] Matthew Wilson et al. Prognostic Significance of Lymph Node Metastases and Ratio in Esophageal Cancer. . J Surg Res. 2008 May 1; 146(1): 11-15.

[8] Refaely Y, Krasna MJ. Multimodality therapy for esophageal cancer. Surg Clin North Am. 2002;82:72946.

[9] Hagen JA, DeMeester SR, Peters JH, Chandrasoma P, DeMeester TR. Curative resection for esophageal adenocarcinoma: Analysis of 100 en bloc esophagectomies. Ann Surg. 2001;234:520-30.

[10] Nigro JJ, DeMeester SR, Hagen JA, DeMeester TR, Peters JH, Kiyabu M, et al. Node status in transmural esophageal adenocarcinoma and outcome after en bloc esophagectomy. J

ThoracCardiovasc Surg. 1999;117:960-8.

[11] Hu Y, Hu C, Zhang H, Ping Y, Chen LQ. How does the number of resected lymph nodes influence TNM staging and prognosis for esophageal carcinoma? Ann Surg Oncol. 2010;17:784-90.

[12] Eloubeidi MA, Desmond R, Arguedas MR, Reed CE, Wilcox CM. Prognostic factors for the survival of patients with esophageal carcinoma in the U.S: The importance of tumor length and lymph node status. Cancer. 2002;95:1434-43.

[13] Nigro JJ, DeMeester SR, Hagen JA, DeMeester TR, Peters JH, Kiyabu M, et al. Node status in transmural esophageal adenocarcinoma and outcome after en bloc esophagectomy. J ThoracCardiovasc Surg. 1999;117:960-8.

[14] Matthew Wilson, B.A, et al. Prognostic Significance of Lymph Node Metastases and Ratio in Esophageal Cancer. J Surg Res. 2008 May 1; 146(1): 11-15.

[15] He Z, Wu S, Li Q, Lin Q, Xu J (2013) Use of the Metastatic Lymph Node Ratio to Evaluate the Prognosis of Esophageal Cancer Patients with Node Metastasis Following Radical Esophagectomy. PLoS ONE 8(9): e73446. doi:10.1371/journal.pone.0073446.

[16] Feng JF, Huang Y, Chen L, Zhao Q. Prognostic analysis of esophageal cancer in elderly patients: Metastatic lymph node ratio versus 2010 AJCC classification by lymph nodes. World J Surg Oncol. 2013;11:162.

[17] Chen LQ1, Hu CY, Zhang HL, He M, Meng XL, Ping YM. Influence of number of removed lymph nodes on the TNM staging and survival in advanced esophageal carcinoma. ZhonghuaZhong LiuZaZhi. 2007 Aug;29(8):604-8.

[18] Greenstein AJ, Litle VR, Swanson SJ, Divino CM, Packer S, Wisnivesky JP .Prognostic significance of the number of lymph node metastases in esophageal cancer. J Am Coll Surg. 2008 Feb;206(2):239-46.

[19] Greenstein AJ1, Litle VR, Swanson SJ, Divino CM, Packer S, Wisnivesky JP. Effect of the number of lymph nodes sampled on postoperative survival of lymph node-negative esophageal cancer. Cancer. 2008 Mar 15;112(6):1239-46.

Volume 6 Issue 12, December 2017 www.ijsr.net 